\section{8 \\ RACIAL DISPARITIES IN PATIENTS WITH COVID-19 INFECTION AND GYNECOLOGIC MALIGNANCY}

${ }^{1}$ Olivia Lara, ${ }^{2}$ Maria Smith, ${ }^{3}$ Yuyan Wang, ${ }^{4}$ Roisin O'cearbhaill, ${ }^{5}$ Stephanie Blank, ${ }^{6}$ Anne Knisely, ${ }^{7}$ Jennifer Mceachron, ${ }^{8}$ Lisa Gabor, ${ }^{9}$ Eloise Chapman-Davis, ${ }^{4}$ Justin Jee, ${ }^{8}$ Sara Isani, ${ }^{3}$ Mengling Liu, ${ }^{6}$ Jason Wright, ${ }^{1}$ Bhavana Pothuri. ${ }^{1}$ Nyu Langone Health; Obstetrics and Gynecology; ${ }^{2}$ NYU Langone Health, New York, United States; ${ }^{3}$ Nyu Langone Health; Population Health; ${ }^{4}$ Memorial Sloan Kettering Cancer Center; Weill Cornell Medical College; Medical Oncology; ${ }^{5} / \mathrm{cahn}$ School of Medicine at Mount Sinai; Obstetrics, Gynecologic and Reproductive Science; ${ }^{6}$ College of Physicians and Surgeons, Columbia University; Obstetrics and Gynecology; ' 5 State University of New York Downstate Medical Center; Obstetrics and Gynecology; ${ }^{8}$ Montefiore Medical Center and Albert Einstein College of Medicine; Obstetrics and Gynecology and Women's Health; ${ }^{9}$ Cornell University; Obstetrics and Gynecology

\subsection{6/ijgc-2020-ESGO.96}

Introduction/Background Mounting evidence suggests disproportionate COVID-19 hospitalizations and deaths due to racial disparities. The association of race in a cohort of gynecologic oncology patients with SARS-CoV-2 infection is unknown.

Methodology Data were abstracted from gynecologic oncology patients with COVID-19 infection among 8 New York City (NYC) area hospital systems. Multivariable mixed-effects logistic regression model accounting for county clustering was utilized to analyse COVID-19 related hospitalization and mortality.

Results Of 193 patients with gynecologic cancer and COVID19, 67 (34.7\%) were Black and 126 (65.3\%) were non-Black. Black patients were more likely to require hospitalization compared with non-Black patients $(71.6 \%$ [48/67] vs. 46.0\% [58/ 126], $\mathrm{P}=.001)$. Of $34(17.6 \%)$ patients who died from COVID-19, 14 (41.2\%) were Black. Among those hospitalized, Black patients compared to non-Black patients were more likely to: have $\geq 3$ comorbidities $(81.1 \%$ [30/37] vs $59.2 \%$ [29/49], $\mathrm{P}=.05)$; reside in Brooklyn $(81.0 \%$ [17/21] vs $44.4 \%$ $[12 / 27], \mathrm{P}=.02)$; live with family $(69.4 \%$ [25/36] vs $41.6 \%$ $[37 / 89], \mathrm{P}=.009)$; and have public insurance $(79.6 \%$ [39/49] vs $53.4 \%$ [39/73], $\mathrm{P}=.006)$. In multivariable analysis, for patients younger than 65 years of age, Black patients were more likely to require hospitalization compared to non-Black patients (OR, 4.87; 95\% CI 1.82 to $12.99, \mathrm{P}=.002)$.

Conclusion Although Black patients with gynecologic cancer represented only $1 / 3$ of patients, they accounted for disproportionate rates of hospitalization $(>45 \%)$ and death $(>40 \%)$ due to COVID-19 infection; younger Black patients had nearly 5-fold greater risk of hospitalization. Efforts to understand and improve these disparities in COVID-19 outcomes in Black patients are critical.

Disclosures B.P. reports grants, personal fees and non-financial support outside the submitted work; institutional PI for industry sponsored trials from Tesaro/GSK, AstraZeneca, Merck, Genentech/Roche, and Clovis Oncology. Compensated advisory boards include Tesaro/GSK, AstraZeneca, Merck and Eisai. J.J. reports a patent license from MDSeq Inc. R.OC reports personal fees from Tesaro, GlaxoSmithKline, Regeneron and Genentech USA, outside the submitted work and non-compensated steering committee member for the PRIMA, Moonstone (Tesaro/GSK) and DUO-O (AstraZeneca) studies. R.OC's institute receives funding for clinical research from Celgene/Juno, Tesaro/GSK, Ludwig Cancer Institute, Abbvie, Regeneron, TCR2 Therapeutics, Atara Biotherapeutics, Marker Therapeutics, Syndax Pharmaceuticals, Genmab Therapeutics, Sellas Therapeutics, Genentech, Kite Pharma, Gynecologic Oncology Foundation. S.V.B. has research collaborations with Roche/Genentech, Tesaro/GK, Seattle Genetics, Merck and Asta Zeneca

\section{Ovarian cancer}

\section{DIAGNOSTIC ACCURACY OF SERUM INSULIN-LIKE GROWTH FACTOR BINDING PROTEIN 2 FOR OVARIAN CANCER}

Pande Kadek Aditya Prayudi, I Nyoman Gede Budiana, Ketut Suwiyoga. Division of Gynecologic Oncology, Department of Obstetrics and Gynecology Faculty of Medicine Udayana University/Sanglah General Hospital

\subsection{6/ijgc-2020-ESG0.97}

Introduction/Background Insulin-like growth factor binding protein-2 (IGFBP2) have been shown to play important roles in the pathogenesis of ovarian cancer. It also serves as a potential biomarker for prognosis of ovarian cancer. However, its role in the diagnosis of ovarian cancer has never been studied. This study is aimed to determine the diagnostic accuracy of serum IGFBP2 in differentiating between malignant and benign ovarian lesion.

Methodology Preoperative IGFBP2 level was determined from the serum of 76 patients with adnexal mass who underwent exploratory laparotomy and subsequent histopathology examination at Sanglah General Hospital, Denpasar, Bali, Indonesia. Diagnostic accuracy of IGFPB2 level was determined from the receiver operating characteristics curve (ROC).

Results Of the 76 patients, 46 patients were diagnosed with ovarian cancer and 30 patients were diagnosed with benign ovarian lesions. Serum IGFBP2 level was significantly higher in patients with ovarian cancer, as compared to those with benign ovarian lesions (median: 945.9 vs. $401.5 \mathrm{~g} / \mathrm{ml}$, $\mathrm{p}<0.001$ ). Using a cut off value of $551.6 \mathrm{ng} / \mathrm{ml}$, the area under the ROC (AUC) for diagnosing ovarian cancer was $0.815 \quad(95 \%$ CI $0.721-0.910, \quad \mathrm{p}<0.001)$, sensitivity was $76.1 \%$, specificity was $80 \%$, and diagnostic odd ratio (DOR) was 12.7 (95\% CI 4.1-39.0, $\mathrm{p}<0.001)$. The diagnostic performance of IGFBP2 was enhanced in postmenopausal women [AUC 0.893 (95\% CI $0.771-1.000, \mathrm{p}=0.002$ ), sensitivity $85 \%$, specificity $85.7 \%$, DOR 34 (95\% CI 2.9-392.8), $\mathrm{p}=0.001]$ and in advance stage [AUC 0.904 (95\% CI $0.806-1.000$, $\mathrm{p}<0.001)$, sensitivity $87.5 \%$, specificity $80 \%$, DOR $28 \quad(95 \%$ CI 6.2-126), $\mathrm{p}<0.001]$.

Conclusion IGFBP2 is a potential biomarker for diagnosis of ovarian cancer.

Disclosures Pande Kadek Aditya Prayudi discloses no potential conflict of interest. I Nyoman Gede Budiana discloses no potential conflict of interest. Ketut Suwiyoga discloses no potential conflict of interest.

\section{ROLE OF MICRORNA-145 IN EPITHELIAL OVARIAN CANCER}

${ }^{1}$ Carmen Romero, ${ }^{1}$ Maritza P Garrido, ${ }^{1}$ Ignacio Torres, ${ }^{1}$ Andrea Hernandez, ${ }^{2}$ Jonas Chnaiderman, ${ }^{1}$ Margarita Vega, ${ }^{1}$ Alberto Selman, ${ }^{3}$ Jessica Preisler, ${ }^{4}$ Lorena Lobos. ${ }^{1}$ Hospital Clínico Universidad de Chile; ${ }^{2}$ Instituto de Ciencias Biomédicas, Facultad de Medicina, Universidad de Chile; ${ }^{3}$ Hospital Clínico Universidad de Chile; Clínica Alemana de Santiago; ${ }^{4}$ Centro De Medicina Regenerativa, Facultad de Medicina, Universidad del Desarrollo

\subsection{6/ijgc-2020-ESGO.98}

Introduction/Background Epithelial ovarian cancer (EOC) is one of the deadliest gynaecologic malignancies worldwide. Nerve Growth Factor (NGF) and its high affinity receptor 
TRKA increase during epithelial ovarian cancer progression, promoting cell proliferation and angiogenesis, by increasing several oncogenic proteins. Many reports have showed that microRNA-145 (miR-145) is down-regulated in EOC, promoting the increase of oncoproteins. Our aims of were to evaluate whether NGF changes the miR-145 levels in EOC cells and the role of this $\mathrm{miR}$ in vitro and in vivo experiments.

Methodology The human tissues and blood samples were obtained with informed consent and approved by Ethic Committee of our Institution. MicroRNA-145 levels were measured by qPCR in EOC samples, EOC cells lines (A2780 and SKOV3) and in serum samples. NGF action on miR-145 levels in EOC cell lines were evaluated using an inhibitor of TRKA (high affinity receptor of NGF). The over-expression of miR145 in EOC cells were done by lipofectamine 2000 and by pGPGLenti-CMV-GFP vector. As control we also used the empty vector. After the transfection assay it was evaluated the migration and invasion of A2780 and SKOV3 cells. After stable miR over-expression assays, the GFP-expressing cells were sorted using GFP mark and the in vivo assays were done. Animal studies were conducted in accordance with appropriate guidelines of Ethical Committee of the Institution. Two groups of Immune-compromised NOD/SCID mice were injected with 8 x106 cells (intraperitoneal and subcutaneous) of A2780 or SKOV3 cells.

Results NGF decreased the miR-145 levels in A2780 and SKOV3 cell lines. Also, when an inhibitor of TRKA receptor was used, levels of this miR increased. Beside, miR-145 levels decreased with the EOC progression in both tissues and plasma samples. MicroRNA-145 over-expression in EOC cells decreased cell proliferation and invasion. Also, the stable overexpression of miR-145 in EOC cells lines decreased malignant ascites presence in the animals. On the other hand, a delay in the tumour formation was observed in EOC cells which overexpress $\mathrm{miR} 145$ in subcutaneous lateral flank compared with the mice injected with control EOC cells.

Conclusion These results suggest that overexpression/delivery of miR-145 could be a future adjuvant therapy in epithelial ovarian cancer

Disclosures The authors declare that there is no conflict of interest. This work was funded by Fondo Nacional de Desarrollo Científico y Tecnológico (FONDECYT) No 1160139 to CR

\section{RISK FACTORS FOR LYMPH NODE METASTASIS OF OVARIAN, FALLOPIAN TUBE AND PRIMARY PERITONEAL CANCER IN HEREDITARY BREAST AND OVARIAN CANCER SYNDROME}

${ }^{1}$ Takashi Mitamura, ${ }^{2}$ Masayuki Sekine ${ }^{3}$ Masami Arai, ${ }^{4}$ Shiro Yokoyama, ${ }^{5}$ Hiroko Yamashita, ${ }^{5}$ Hidemichi Watari, ${ }^{5}$ Ichiro Yabe, ${ }^{6}$ Hiroyuki Nomura, ${ }^{2}$ Takayuki Enomoto, ${ }^{4}$ Seigo Nakamura. ${ }^{1}$ Hokkaido University Hospital; ${ }^{2}$ Niigata University Graduate School of Medical and Dental Science; ${ }^{3}$ Juntendo University, Graduate School of Medicine; ${ }^{4}$ Showa University School of Medicine; ${ }^{5}$ Hokkaido University Hospital; ${ }^{6}$ Fujita Health University

\subsection{6/ijgc-2020-ESG0.99}

Introduction/Background To establish an individualized surgical strategy for ovarian cancer in patients with the germline BRCA1 and BRCA2 pathogenic variants (BRCA1+ and BRCA2+), we investigated the clinicopathological characteristics that are involved in the increased risk of lymph node metastasis (LNM).

Methodology We retrospectively reviewed the data of 509 Japanese women who underwent BRCA1 and BRCA2 genetic testing and were registered in the database of the Japanese HBOC Consortium.

Results BRCA1+ and BRCA2 + were found in 232 (45.6\%) of patients in our database, while other patients had no pathogenic variant (BRCA-). The prevalence of LNM was not markedly different between BRCA- (19.5\%), BRCA1+ $(18.4 \%)$ and BRCA2+ (26.2\%). Multivariate analysis revealed an absence of a family history of ovarian cancer as an independent predictor for an increased risk of LNM in BRCA1+, and the prevalence of LNM was $11.7 \%$ and $42.0 \%$ in the groups with and without a family history of ovarian cancer, respectively. This subgroup without a family history of ovarian cancer did not show any correlation with a particular variant of BRCA1.

Conclusion This study suggested that the existence of a subgroup in which the absence of a family history of ovarian cancer was an independent predictor for an increased risk of lymph node metastasis and certain genetic factors related to the penetrance of hereditary breast and ovarian cancer syndrome might alter the frequency of LNM in BRCA1+ ovarian cancer. We will need to investigate whether or not the same surgical strategy for lymphadenectomy as BRCA- is suitable for this BRCA1+ subgroup.

Disclosures There are no conflict of interest disclosures from any authors.

\section{CONDITIONAL RELATIVE SURVIVAL OF OVARIAN CANCER : A KOREAN NATIONAL CANCER REGISTRY STUDY}

${ }^{1}$ Jaeman Bae, ${ }^{2}$ Dong Wook Shin, ${ }^{3}$ Kyu-Won Jung. ' Hanyang University College of Medicine; Department of Gynecologic Oncology; ${ }^{2}$ Department of Family Medicine, Samsung Medical Center; Sungkyunkwan University School of Medicine; ${ }^{3}$ The Korea Central Cancer Registry, National Cancer Center; 323 Ilsan-Ro, Ilsandong-Gu, Goyang-Si, Gyeonggi-Do

\subsection{6/ijgc-2020-ESG0.100}

Introduction/Background Conditional relative survival (CRS) considers changes in prognosis over time and thus, may offer more useful estimates for survivors and clinicians. We aimed to investigate the CRS among patients with ovarian cancer with comprehensive stratification by various factors that influence survival probabilities.

Methodology This nationwide retrospective cohort study used data from the Korean Central Cancer Registry. We included 78,606 patients diagnosed with cervical cancer as their first cancer between January 1, 1997 and December 31, 2016. CRS and the conditional probabilities of death for the following 1 year were calculated stratified by age at diagnosis, histology, stage at diagnosis, year of diagnosis, and social deprivation index.

Results The 5-year relative survival rate at the time of diagnosis was $61.1 \%$ for all cases. The probability of surviving an additional 5 years conditioned on having already survived 1 , $2,3,4$, and 5 years after diagnosis was $65.0 \%, 69.5 \%$, $74.6 \%, 79.3 \%$, and $83.9 \%$. Patients with poorer initial survival estimates (older, advanced stage) generally showed the 